\title{
Concerns among people who use opioids during the COVID-19 pandemic: a natural language processing analysis of social media posts
}

Abeed Sarker $^{1 *}$, Nisha Nataraj ${ }^{2}$, Wesley Siu ${ }^{3}$, Sabrina Li ${ }^{4}$, Christopher M. Jones ${ }^{2}$ and Steven A. Sumner ${ }^{2}$

\begin{abstract}
Background: Timely data from official sources regarding the impact of the COVID-19 pandemic on people who use prescription and illegal opioids is lacking. We conducted a large-scale, natural language processing (NLP) analysis of conversations on opioid-related drug forums to better understand concerns among people who use opioids.

Methods: In this retrospective observational study, we analyzed posts from 14 opioid-related forums on the social network Reddit. We applied NLP to identify frequently mentioned substances and phrases, and grouped the phrases manually based on their contents into three broad key themes: (i) prescription and/or illegal opioid use; (ii) substance use disorder treatment access and care; and (iii) withdrawal. Phrases that were unmappable to any particular theme were discarded. We computed the frequencies of substance and theme mentions, and quantified their volumes over time. We compared changes in post volumes by key themes and substances between pre-COVID-19 (1/1/20192/29/2020) and COVID-19 (3/1/2020-11/30/2020) periods.

Results: Seventy-seven thousand six hundred fifty-two and 119,168 posts were collected for the pre-COVID-19 and COVID-19 periods, respectively. By theme, posts about treatment and access to care increased by $300 \%$, from 0.631 to 2.526 per 1000 posts between the pre-COVID-19 and COVID-19 periods. Conversations about withdrawal increased by $812 \%$ between the same periods ( 0.026 to 0.235 per 1,000 posts). Posts about drug use did not increase ( 0.219 to 0.218 per 1,000 posts). By substance, among medications for opioid use disorder, methadone had the largest increase in conversations (20.751 to 56.313 per 1,000 posts; $171.4 \%$ increase). Among other medications, posts about diphenhydramine exhibited the largest increase (0.341 to 0.927 per 1,000 posts; $171.8 \%$ increase).
\end{abstract}

Conclusions: Conversations on opioid-related forums among people who use opioids revealed increased concerns about treatment and access to care along with withdrawal following the emergence of COVID-19. Greater attention to social media data may help inform timely responses to the needs of people who use opioids during COVID-19.

\footnotetext{
*Correspondence: abeed@dbmi.emory.edu

1 Department of Biomedical Informatics, School of Medicine, Emory

University, GA 30322 Atlanta, Georgia

Full list of author information is available at the end of the article

Disclaimer The findings and conclusions in this report are those of

the authors and do not necessarily represent the official position of the

Centers for Disease Control and Prevention.
}

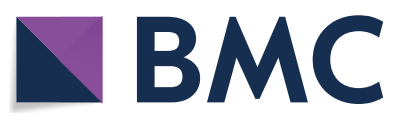

(s) 2022. Open Access This article is licensed under a Creative Commons Attribution 4.0 International License, which permits use, sharing, adaptation, distribution and reproduction in any medium or format, as long as you give appropriate credit to the original author(s) and the source, provide a link to the Creative Commons licence, and indicate if changes were made. The images or other third party material in this article are included in the article's Creative Commons licence, unless indicated otherwise in a credit line to the material. If material is not included in the article's Creative Commons licence and your intended use is not permitted by statutory regulation or exceeds the permitted use, you will need to obtain permission directly from the copyright holder. To view a copy of this licence, visit http://creativecommons.org/licenses/by/4.0/. The Creative Commons Public Domain Dedication waiver (http://creativeco mmons.org/publicdomain/zero/1.0/) applies to the data made available in this article, unless otherwise stated in a credit line to the data. 
Keywords: Opioids (MeSH ID: D000701), Opioid use disorder (MeSH ID: D009293), COVID-19 (MeSH ID: D000086382), Coronavirus (MeSH ID: D017934), Natural language processing (MeSH ID: D009323), Text mining (MeSH ID: D057225), Social media (MeSH ID: D061108)

\section{Background}

As of June 2021, the novel coronavirus disease (COVID19) pandemic has claimed the lives of over 590,000 individuals in the US and 3.8 million globally [1]. Public health experts suggest that the intersection of the COVID-19 pandemic and the ongoing drug overdose epidemic has had detrimental impacts on efforts to reduce deaths $[2,3]$. Estimates in the US show that monthly increases in overdose deaths during April and May 2020, the early months of the pandemic, were the largest since provisional data reporting began in 2015 [4].

Persons with substance use disorder (SUD) are thought to be directly at a higher risk for COVID-19 and poor outcomes because of higher rates of immunocompromised status and other underlying comorbidities [3]. Indirectly, disruptions in access to SUD treatment and mental health services have made it more difficult for patients to receive timely and consistent care [5], and social distancing measures have resulted in individuals using substances alone, raising risk for overdose death $[6$, 7]. There are additional concerns that housing, employment, and economic instability, correlates of substance use problems, have substantially increased during the pandemic $[8,9]$.

However, little is known about immediate concerns among communities of people who use prescription and illegal opioids, and how those concerns have evolved during the COVID-19 pandemic. Our goal was to harness large-scale, publicly-available conversation data from posts made on Reddit, which houses the largest online opioid-related drug forums, to better understand how communities of people who use opioids have been impacted by the pandemic. We use natural language processing (NLP) and a mixed-methods design to study trends in topics prior to and since the emergence of the COVID-19 pandemic. Improved, timely awareness of issues affecting people who use opioids during the pandemic can help local, state, and federal organizations better respond to the needs of these communities in the moment.

\section{Methods}

\section{Data source}

Reddit, one of the largest social networks and the largest online forum with over 52 million daily users, hosts active community forums on a range of substance-use related topics including, but not limited to, opioid use and has been extensively used for similar research [10]. We identified 14 forums (i.e., "subreddits") containing discussions on prescription and illegal opioid use and recovery. We retrieved historical public post data using the Python-Reddit Application Programming Interface Wrapper. ${ }^{1}$ The Emory Institutional Review Board determined this secondary research using publicly available information exempt from review. We divided historical posts in the subreddits into two data subsets to study any changes in conversation following the emergence of the pandemic:

- pre-COVID-19 period: January 1, 2019-February 29, 2020.

- COVID-19 period: March 1, 2020-November 30, 2020.

\section{Data processing}

The included subreddits span topics ranging from opioid use disorder (OUD) recovery strategies to personal experiences with opioids. Consistent with standard convention in NLP research, posts were initially preprocessed by converting text to lowercase, reducing words to their stems (i.e., stemming), and removing stopwords (e.g., 'a,' 'the', 'in', etc.) using the Python Natural Language Toolkit. $^{2}$

Next, potential phrases of interest were identified quantitatively by calculating:

- Frequency distributions of words/phrases 1-3 tokens long for each study period.

- Term frequency inverse document frequency (TFIDF) values, including monthly and aggregate TFIDF values for both periods. TF-IDF calculations assign weights to a given word/phrase by frequency of appearance in a data subset relative to posts from the remaining dataset. We employed a customized TF-IDF method where "TF" denotes the term frequency for a given period (i.e., month) and "IDF" denotes "the inverse of the term frequency" from other periods (Supplementary material A).

\footnotetext{
${ }^{1}$ Boe B. PRAW: The Python Reddit API Wrapper. (https://praw.readthedocs. io/en/latest/). Accessed: 06/21/2021.

${ }^{2}$ https://www.nltk.org/. Accessed: 06/21/2021.
} 


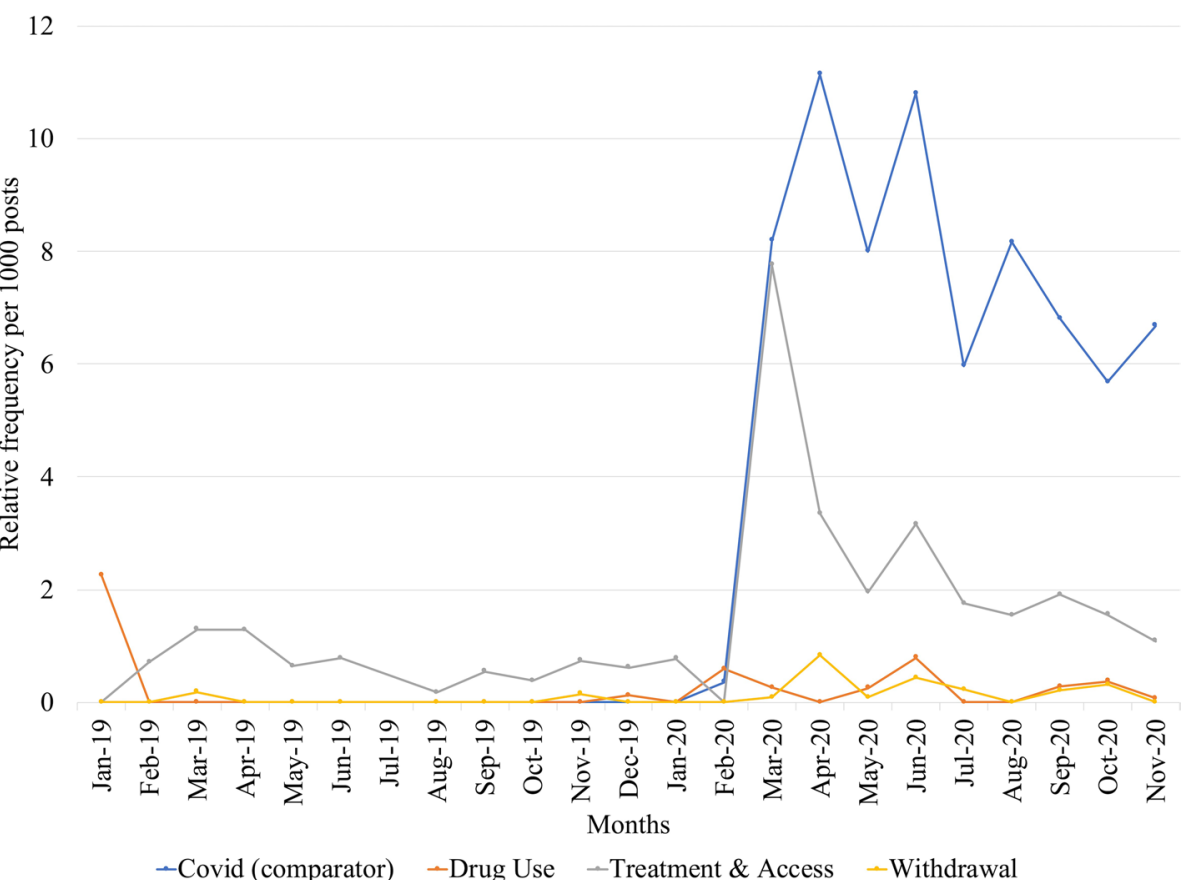

Fig. 1 Month-to-month relative frequencies for expressions belonging to the themes (i) drug use, (ii) treatment and access, and (iii) withdrawal. Relative frequencies for the term 'covid' are provided for comparison

Subject matter experts in substance use (CMJ and SAS) then manually reviewed the phrases of interest, combined with their spelling and phrasing variants, to identify the top 50 unique terms relevant to substance use (Supplementary material B). These were then used to explore two main questions: how did the emergence of COVID-19 shift 1) substance-use related themes being discussed? and 2) conversations around specific substances?

\section{Shifts in substance use related themes}

The subject matter experts grouped the selected terms into three broad themes by primary content discussed: 1) prescription and illegal opioid use, 2) SUD treatment and access to care, and 3) withdrawal. Relative frequencies were then calculated by month for each theme using the total monthly number of posts from the 14 included subreddits as the denominator. We also plotted trends in the frequency of the term 'COVID'. Since this term was understandably absent before the pandemic, its rise following the outbreak served as a suitable comparison for other substance-related themes explored. Qualitative review of posts by theme was conducted to validate our automatic findings and further explore the nature of conversations.

\section{Shifts in specific drug mentions over time}

We also studied changes in the mentions of specific substances in the two periods. Drugs of interest were identified using the aforementioned quantitative and qualitative approaches (Appendices-C-D). We combined brand, generic, and street names for substances into single items, where necessary. Since drug names are often misspelled or expressed using non-standard expressions on social media, we used an automatic variant generator to include commonly-used lexical variants. We then computed the relative frequencies of these substances in the pre-COVID-19 and COVID-19 periods to explore trends. All analyses were conducted in Python (Version 3.8).

\section{Results}

Posts were collected and analyzed across the 14 opioidrelated subreddits, with 77,652 and 119,168 posts during the pre-COVID-19 and COVID-19 periods, respectively. Figure 1 shows the monthly relative frequencies of the major themes of drug use, treatment and access, and withdrawal per 1,000 posts. For comparison, "COVID" was rarely mentioned before March 2020, but its relative frequency rapidly rose once the pandemic received widespread attention. Of all themes, there was a considerable increase in posts about treatment and access 
Table 1 Change in post-volume pre-COVID-19 and COVID-19 periods among leading substances discussed on opioid-related forums

\begin{tabular}{|c|c|c|c|c|}
\hline & $\begin{array}{l}\text { Pre-COVID-19 period daily } \\
\text { average }\end{array}$ & $\begin{array}{l}\text { COVID-19 period daily } \\
\text { average }\end{array}$ & Difference & Change (\%) \\
\hline \multicolumn{5}{|c|}{ Medications for Opioid Use Disorder and Overdose Reversal } \\
\hline Methadone & 20.751 & 56.313 & 35.562 & $171.4 \%$ \\
\hline Naltrexone & 1.087 & 2.451 & 1.364 & $125.5 \%$ \\
\hline Buprenorphine & 18.426 & 36.225 & 17.800 & $96.6 \%$ \\
\hline Naloxone & 1.680 & 3.116 & 1.436 & $85.5 \%$ \\
\hline \multicolumn{5}{|c|}{ Other Substances used in Non-Medical Settings } \\
\hline Iboga & 0.520 & 1.175 & 0.655 & $125.9 \%$ \\
\hline Kratom & 3.454 & 7.222 & 3.768 & $109.1 \%$ \\
\hline \multicolumn{5}{|l|}{ Prescription and Illegal Opioids } \\
\hline Hydrocodone & 0.986 & 2.687 & 1.701 & $172.6 \%$ \\
\hline Fentanyl & 9.506 & 25.404 & 15.898 & $167.2 \%$ \\
\hline Oxycodone & 2.191 & 5.796 & 3.606 & $164.6 \%$ \\
\hline Other prescription opioids & 1.605 & 3.687 & 2.083 & $129.8 \%$ \\
\hline Heroin & 8.325 & 14.677 & 6.352 & $76.3 \%$ \\
\hline Carfentanil & 0.325 & 0.411 & 0.086 & $26.5 \%$ \\
\hline \multicolumn{5}{|l|}{ Other Medications } \\
\hline Diphenhydramine ${ }^{a}$ & 0.341 & 0.927 & 0.586 & $171.8 \%$ \\
\hline Diazepam & 0.504 & 1.324 & 0.820 & $162.9 \%$ \\
\hline Other benzodiazepines & 4.791 & 11.178 & 6.388 & $133.3 \%$ \\
\hline Alprazolam & 1.384 & 3.164 & 1.780 & $128.7 \%$ \\
\hline Dextromethorphan ${ }^{a}$ & 0.146 & 0.291 & 0.145 & $99.4 \%$ \\
\hline Dextroamphetamine & 1.252 & 2.131 & 0.879 & $70.2 \%$ \\
\hline
\end{tabular}

Substances are ordered by percentage change within each medication grouping. Category of "other opioids" and "other benzodiazepines" represents all such medications not explicitly mentioned in the table. Counts are based on generic and trade names, and their common misspellings

a Denotes substances available in over-the-counter forms

to care following the emergence of the pandemic which increased $300 \%$ from 0.631 in the pre-COVID-19 period to 2.526 per 1,000 posts. Posts concerning this theme were most prevalent during March 2020, accounting for approximately 8 out of 1,000 posts. Conversations about withdrawal also increased in the COVID-19 period, albeit smaller in scale compared to treatment and access conversations, exhibiting an $812 \%$ rise from 0.026 to 0.235 per 1,000 posts. Posts about drug use did not increase during the COVID-19 period (0.219 to 0.218 per 1000 posts; $-0.001 \%$ change).

Table 1 presents an examination of changes in the average daily frequency of specific substances mentioned. There was a consistent increase in mentions of opioids and certain other substances. Methadone, a medication for opioid use disorder, demonstrated the largest increase (20.751 to $56.313 ; 171.4 \%)$. Among other medications, diphenhydramine experienced the largest relative increase in mentions on opioid-related forums, followed by benzodiazepines. Kratom and Iboga, other substances not used in medical settings, also had increases.

Qualitative assessment of posts that contained treatment and access to care related content revealed that the majority of posts were from individuals currently in recovery. A large volume of conversations focused on take-home access to methadone. For example, one post (abbreviated for clarity and anonymity) stated: ... "The most [take-homes] one can get at [my opioid treatment program] is 1 week. I asked if they would have to make special exceptions because of the [COVID-19] crisis. I was told that nobody has to do anything for us... I understand that take home medications could kill kids, pets, etc.[.] if someone is irresponsible[,] but I think they should let up a little bit." Other posts highlighted changes opioid treatment programs had made since the onset of the pandemic: "... I usually have 2 week [takehomes], I'm getting 28 days now [because] of COVID19. My clinic has ONLY been testing unstable patients, new patients, or ones with recently failed [urinalyses]. But all of it is up to the individual clinics or corporate owners of the clinic.". Additional examples are available in Supplementary material $\mathrm{E}$. 


\section{Discussion}

This study used large-scale real-world conversation data to explore the concerns of people who use opioids after the emergence of the COVID-19 pandemic. Our mixed-methods approach reveals that conversations about treatment and access to care increased markedly during COVID-19, with a focus on methadone access and increased discussion of over-the-counter medications (e.g., diphenhydramine) and other substances (e.g., kratom).

There is increased demand for timely data to guide public health prevention activities as health professionals have raised several potential concerns facing SUD treatment and overdose prevention arising from the COVID-19 pandemic [11, 12]. Leading sources of information on drug use traditionally include large-scale surveys such as the National Survey on Drug Use and Health and data derived from toxicology testing of overdose deaths [13]. Unfortunately, due to time-consuming nature of these data collection methodologies, real-time data from these official sources are not available to guide decision making. Consistent with other studies [14, 15], our results highlight the role and relevance of novel data sources to explore early insights and complement traditional data systems.

Manual examination of posts verified that the NLP analysis findings are indeed informative. We found large increases in conversations about treatment and access to care from March to November 2020. For methadone, a considerable amount of the increase concerned regional policies about medications for opioid use disorder access. In March 2020, the Substance Abuse and Mental Health Services Administration released guidance allowing opioid treatment programs to provide take-home doses of methadone and buprenorphine for established, stable patients. Under access to care, discussions surrounding methadone increased the most relative to other medications for opioid use disorders. Interestingly, while policy changes attempted to facilitate access to medications for opioid use disorder during COVID-19, access to methadone was under stricter regulation than buprenorphine; for example, clinicians at opioid treatment programs could initiate buprenorphine remotely for new patients without an in-person examination unlike methadone initiation which still required in-person examinations. Additionally, in office-based settings, clinicians with a Drug Addiction Treatment Act waiver to prescribe buprenorphine for OUD were provided emergency authority to initiate new patients remotely without inperson examinations.

Despite considerable speculation about how COVID19 is shifting substance-use markets and patterns, we found that conversations among people who use opioids were overwhelmingly focused on treatment relative to conversations about procuring and using illegal substances. This underscores the interest in medications for opioid use disorder, despite the myriad challenges that face the medications for opioid use disorder maintenance, especially during the pandemic. We note that our analysis did reveal an increase in conversations about fentanyl during the COVID-19 period when overdose deaths involving synthetic opioids such as illegally made fentanyl accelerated [16].

Non-opioid substances showed marked increases in conversations within the opioid subreddits, the largest of which were seen for over-the-counter (e.g., diphenhydramine) or prescription (e.g., benzodiazepines) medications used to treat symptoms, including those associated with withdrawal [17]. Of note, benzodiazepines have been known to be co-ingested with opioids, often with fatal consequences [18]. Kratom, which is unregulated, and iboga (ibogaine), a schedule-I controlled substance also showed increases in conversations. These substances have been of interest to people who use opioids as alternatives for opioid treatment or withdrawal in non-medical settings and have received widespread social media attention [19] despite concerns about safety [20, 21]. Insights from our work may help inform population-level monitoring efforts for drug poisoning and overdose education by providers and public health practitioners, specifically in the context of COVID-19.

This work has some limitations. Although the data sources employed represent large-scale data from the largest drug forums online, we recognize that such data are a convenience sample and may not fully represent concerns of populations who do not use these platforms. Additionally, stay-at-home orders could have resulted in conversations about the studied themes shifting from in-person to online channels. Thus, results from these efforts should be used to help generate early hypotheses and insights that can be further assessed using traditional health and substance use surveillance systems. Furthermore, this study uses qualitative review of posts; future work should evaluate the accuracy of fully-automated, unsupervised machine learning methods to further accelerate the timeliness of analyses and insights.

Nevertheless, our findings show that treatment and access to care continue to be major themes discussed by people who used opioids during the COVID-19 pandemic. Rapidly identifying and addressing concerns of these communities through novel data and analytic methodologies are crucial. Removing barriers to care, including through the expansion of telehealth and allowance of take-home doses of medications for opioid use disorder from opioid treatment programs, are important to support overdose prevention activities and long-term 
recovery as part of the overdose public health emergency response.

\section{Conclusion}

Our analysis demonstrates the utility of social media for studying SUD, particularly at times when significant social changes occur, such as the spread of the COVID19 pandemic. There is still considerable uncertainty about how COVID-19 will keep impacting the lives of people. COVID-19 resulted in substantial increases in chatter related to treatment and access, and withdrawal. We found that this was because people suffering from SUD had major concerns about maintaining their treatment once stay-at-home orders were issued. Active surveillance using social media data can help track emerging concerns and problems as the world prepares for post-COVID-19 life. Currently, despite the usefulness of social media for SUD research and surveillance, they have been largely under-utilized. We believe that caregivers and policy makers should put greater attention to social media data, as they may help inform timely responses to the needs of people who use opioids during COVID-19.

\section{Abbreviations}

OUD: Opioid use disorder; SUD: Substance use disorder; NLP: Natural language processing; TF-IDF: Term frequency-inverse document frequency.

\section{Supplementary Information}

The online version contains supplementary material available at https://doi. org/10.1186/s13011-022-00442-w.

Additional file 1. A.TF-IDF equation. B.Top 50 selected terms and phrases. C. Drug names and misspellings. D. Medication names and grouping. E. Sample posts.

\section{Acknowledgements}

None

\section{Authors' contributions}

AS performed data collection and analysis of the data with assistance from WS and SL. NN provided feedback, performed analyses, including the coding of the phrases, and aided in preparing the content for the manuscript. CMJ and SAS provided expertise on the topic and guided the analyses. All authors contributed to the final manuscript.

\section{Funding}

Centers for Disease Control and Prevention (CDC) and Emory University.

\section{Availability of data and materials}

Publicly available data from Reddit was used for this study. Authors do not have the authority to release data beyond the examples in the manuscript and supplementary material. Data can be obtained directly from Reddit via the appropriate application programming interface (API).

\section{Declarations}

Ethics approval and consent to participate

The data used in this study are public and as such it was deemed to be exempt (category 4) by Emory University IRB.

\section{Consent for publication}

Not applicable.

\section{Competing interests}

The authors declare that they have no competing interests.

\section{Author details}

${ }^{1}$ Department of Biomedical Informatics, School of Medicine, Emory University, GA 30322 Atlanta, Georgia. ${ }^{2}$ National Center for Injury Prevention and Control, Centers for Disease Control and Prevention, GA 30341 Atlanta, Georgia. ${ }^{3}$ Rollins School of Public Health, Emory University, GA 30322 Atlanta, Georgia. ${ }^{4}$ Department of Computer Science, Emory University, GA 30322 Atlanta, Georgia.

Accepted: 15 February 2022

Published online: 05 March 2022

\section{References}

1. Centers for Disease Control and Prevention. CDC COVID Data Tracker. Trends Number COVID-19 Cases Deaths US Rep. to CDC, by State/Territory. 2020.https://covid.cdc.gov/covid-data-tracker/\#trends_totalandra tedeathssevendayrate (accessed 19 Feb 2021).

2. Linas BP, Savinkina A, Barbosa C, et al. A clash of epidemics: Impact of the COVID-19 pandemic response on opioid overdose. J Subst Abuse Treat. 2021;120: 108158. https://doi.org/10.1016/j.jsat.2020.108158.

3. Volkow ND. Collision of the COVID-19 and Addiction Epidemics. Ann Intern Med. 2020;173:61-2. https://doi.org/10.7326/M20-1212.

4. CDC Health Alert Network. Increase in Fatal Drug Overdoses Across the United States Driven by Synthetic Opioids Before and During the COVID19 Pandemic. Centers for Disease Control MMWR Office. 2020. https://doi. org/10.15585/mmwr.mm6911a4.

5. Pagano A, Hosakote S, Kapiteni K, et al. Impacts of COVID-19 on residential treatment programs for substance use disorder. J Subst Abuse Treat. 2021;123:108255. https://doi.org/10.1016/j.jsat.2020.108255.

6. Bonn M, Palayew A, Bartlett S, et al. Addressing the Syndemic of HIV, Hepatitis C, Overdose, and COVID-19 Among People Who Use Drugs: The Potential Roles for Decriminalization and Safe Supply. J Stud Alcohol Drugs. 2020;81:556-60. https://doi.org/10.15288/JSAD.2020.81.556.

7. Perri $M$, Kaminski $N$, Bonn $M$, et al. A qualitative study on overdose response in the era of COVID-19 and beyond: how to spot someone so they never have to use alone. Harm Reduct J. 2021;18:1-9. https://doi. org/10.1186/S12954-021-00530-3/METRICS.

8. Dasgupta N, Beletsky L, Ciccarone D. Opioid Crisis: No Easy Fix to Its Social and Economic Determinants. Am J Public Health. 2018;108:182-6. https://doi.org/10.2105/AJPH.2017.304187.

9. Haley DF, Saitz R. The Opioid Epidemic during the COVID-19 Pandemic. JAMA - J Am Med Assoc. 2020;324:1615-7. https://doi.org/10.1001/jama. 2020.18543.

10. Bowen DA, O'Donnell J, Sumner SA. Increases in Online Posts About Synthetic Opioids Preceding Increases in Synthetic Opioid Death Rates: a Retrospective Observational Study. J Gen Intern Med. 2019;34:2702-4. https://doi.org/10.1007/s11606-019-05255-5.

11. Wakeman SE, Green TC, Rich J. An overdose surge will compound the COVID-19 pandemic if urgent action is not taken. Nat Med. 2020;26:81920. https://doi.org/10.1038/s41591-020-0898-0.

12. Alexander GC, Stoller KB, Haffajee RL, et al. An Epidemic in the Midst of a Pandemic: Opioid Use Disorder and COVID-19. Ann Intern Med. 2020;173:57-8. https://doi.org/10.7326/M20-1141.

13. Scholl L, Seth P, Kariisa M, et al. Drug and Opioid-Involved Overdose Deaths - United States, 2013-2017. MMWR Morb Mortal Wkly Rep. 2018;67(5152):1419-27. https://doi.org/10.15585/mmwr.mm675152e1. 
14. Substance Abuse and Mental Health Services Administration. Key substance use and mental health indicators in the United States: Results from the 2018 National Survey on Drug Use and Health. (HHS Publication No. PEP19-5068, NSDUH Series H-54). Rockville, MD: Center for Behavioral Health Statistics and Quality, Substance Abuse and Mental Health Services Administration. Retrieved from https://www.samhsa.gov/data/.

15. Wilson N, Kariisa M, Seth P, et al. Drug and Opioid-Involved Overdose Deaths - United States, 2017-2018. MMWR Morb Mortal Wkly Rep. 2020:69:290-7. https://doi.org/10.15585/mmwr.mm6911a4.

16. Centers for Disease Control and Prevention. Overdose Deaths Accelerating During COVID-19 | CDC Online Newsroom | CDC. 2020.https://www. cdc.gov/media/releases/2020/p1218-overdose-deaths-covid-19.html (accessed 21 Jan 2021).

17. Kosten TR, Baxter LE. Review article: Effective management of opioid withdrawal symptoms: A gateway to opioid dependence treatment. Am J Addict. 2019;28:55-62. https://doi.org/10.1111/ajad.12862.

18. Tori ME, Larochelle MR, Naimi TS. Alcohol or Benzodiazepine Co-involvement with Opioid Overdose Deaths in the United States, 1999-2017. JAMA Netw Open. 2020;3(4):e202361. https://doi.org/10.1001/jaman etworkopen.2020.2361.

19. Chancellor S, Nitzburg G, Hu A, et al. Discovering Alternative Treatments for Opioid Use Recovery Using Social Media. In: Proceedings of the 2019 CHI Conference on Human Factors in Computing Systems - CHI '19. New York: ACM Press; 2019

20. Litjens RPW, Brunt TM. How toxic is ibogaine? Clin Toxicol. 2016;54:297302. https://doi.org/10.3109/15563650.2016.1138226.

21. US Food and Drug Administration. FDA and Kratom |FDA. 2019.https:// www.fda.gov/news-events/public-health-focus/fda-and-kratom (accessed 22 Jun 2021)

\section{Publisher's Note}

Springer Nature remains neutral with regard to jurisdictional claims in published maps and institutional affiliations.

- fast, convenient online submission

- thorough peer review by experienced researchers in your field

- rapid publication on acceptance

- support for research data, including large and complex data types

- gold Open Access which fosters wider collaboration and increased citations

- maximum visibility for your research: over $100 \mathrm{M}$ website views per year

At BMC, research is always in progress.

Learn more biomedcentral.com/submissions 